\title{
Pediatric hydrocephalus outcomes in Lusaka, Zambia
}

\author{
Rebecca A. Reynolds, MD, ${ }^{1-3}$ Arnold Bhebhe, MBChB, ${ }^{2}$ Roxanna M. Garcia, MD, MS, MPH, ${ }^{4}$ \\ Shilin Zhao, PhD, ${ }^{5}$ Sandi Lam, MD, MBA, ${ }^{4}$ Kachinga Sichizya, MBChB, ${ }^{2}$ \\ and Chevis N. Shannon, DrPH, MBA, MPH'1,3
}

\begin{abstract}
${ }^{1}$ Department of Neurological Surgery, Vanderbilt University Medical Center, Nashville, Tennessee; ${ }^{2}$ Department of Neurological Surgery, University Teaching Hospital, Lusaka, Zambia; ${ }^{3}$ Surgical Outcomes Center for Kids, Monroe Carell Jr. Children's Hospital, Nashville, Tennessee; ${ }^{4}$ Department of Neurological Surgery, Northwestern University, Chicago, Illinois; and ${ }^{5}$ Vanderbilt Center for Quantitative Sciences, Vanderbilt University Medical Center, Nashville, Tennessee
\end{abstract}

OBJECTIVE Hydrocephalus is a global disease that disproportionally impacts low- and middle-income countries. Limited data are available from sub-Saharan Africa. This study aims to be the first to describe pediatric hydrocephalus epidemiology and outcomes in Lusaka, Zambia.

METHODS This retrospective cohort study included patients < 18 years of age who underwent surgical treatment for hydrocephalus at Beit-CURE Hospital and the University Teaching Hospital in Lusaka, Zambia, from August 2017 to May 2019. Surgeries included ventriculoperitoneal shunt insertions, revisions, and endoscopic third ventriculostomies (ETVs) with or without choroid plexus cauterization (CPC). A descriptive analysis of patient demographics, clinical presentation, and etiologies was summarized, followed by a multivariable analysis of mortality and 90-day complications.

RESULTS A total of 378 patients met the inclusion criteria. The median age at first surgery was 5.5 (IQR $3.1,12.7)$ months, and $51 \%$ of patients were female $(n=193)$. The most common presenting symptom was irritability $(65 \%, n=$ $247)$, followed by oculomotor abnormalities $(54 \%, n=204)$. Postinfectious hydrocephalus was the predominant etiology $(65 \%, n=226 / 347)$, and $9 \%$ had a myelomeningocele $(n=32 / 347)$. It was the first hydrocephalus surgery for $87 \%$ ( $n$ $=309)$ and, of that group, $15 \%$ underwent ETVICPC $(n=45)$. Severe hydrocephalus was common, with $42 \%$ of head circumferences more than $6 \mathrm{~cm}$ above the 97 th percentile $(n=111)$. The median follow-up duration was $33(I Q R 4,117)$ days. The complication rate was $20 \%(n=76)$, with infection being most common $(n=29)$. Overall, $7 \%$ of the patients died $(n=26)$. Postoperative complication was significantly associated with mortality $\left(X^{2}=81.2, p<0.001\right)$ with infections and CSF leaks showing the strongest association ( $X^{2}=14.6$ and 15.2 , respectively, $\left.p<0.001\right)$. On adjusted multivariable analysis, shunt revisions were more likely to have a complication than ETV/CPC or primary shunt insertions (OR 2.45 [95\% Cl 1.26-4.76], $p=0.008)$, and the presence of any postoperative complication was the only significant predictor of mortality (OR 42.9 [95\% Cl 12.3-149.1], p < 0.001).

CONCLUSIONS Pediatric postinfectious hydrocephalus is the most common etiology of hydrocephalus in Lusaka, Zambia, which is similar to other countries in sub-Saharan Africa. Most children present late with neglected hydrocephalus. Shunt revision procedures are more prone to complication than ETVICPC or primary shunt insertion, and postoperative complications represent a significant predictor of mortality in this population.

https://thejns.org/doi/abs/10.3171/2020.5.PEDS20193

KEYWORDS hydrocephalus; Zambia; sub-Saharan Africa; shunt; spina bifida; surgical outcomes

$\mathrm{W}$ HILE the diagnostic criteria of hydrocephalus are similar regardless of country or continent, the etiologies and surgical outcomes of hydrocephalus differ greatly between high-income countries (HICs) and low- and middle-income countries (LMICs). Zambia is a nation with 17 million people in southern, sub-Saharan Africa. ${ }^{1}$ Fifty-seven percent of the population lives below the inter- national poverty line on less than $\$ 1.90$ per person per day, which contributes to its status as an LMIC according to the World Bank..$^{1,2}$ Neurosurgeons are in short supply. According to the last published survey by the World Federation of Neurosurgical Societies (WFNS) in 2016, Zambia reported 1 neurosurgeon to 228,000 people, compared with 1 to 62,000 in the US. ${ }^{3}$ The ratio in Zambia has dramatically improved

ABBREVIATIONS CPC = choroid plexus cauterization; ETV = endoscopic third ventriculostomy; HIC = high-income country; LMIC = low- and middle-income country; PIH = postinfectious hydrocephalus; VP = ventriculoperitoneal.

SUBMITTED March 17, 2020. ACCEPTED May 21, 2020.

INCLUDE WHEN CITING Published online September 11, 2020; DOI: 10.3171/2020.5.PEDS20193. 
since the initial survey circulated by the WHO and WFNS in 2001, citing a ratio of 1 neurosurgeon to 3.6 million people. ${ }^{4}$ New neurosurgical training programs are helping to close this gap. ${ }^{5}$ In 2018, the first Zambian neurosurgery residency training program was launched to address the surgeon shortage; however, the program is young, and it will take time to build enough capacity to adequately address research and clinical disparities.

Hydrocephalus is primarily a disease of childhood in LMICs. It is the most common condition treated in general pediatric neurosurgical practices worldwide. ${ }^{6}$ Hydrocephalus has a particularly high density in sub-Saharan Africa when compared with higher-income settings in northern Africa and around the world. ${ }^{7}$ It is estimated that the incidence of hydrocephalus in LMICs is nearly double that in HICs. ${ }^{8}$ This region suffers disproportionally from higher rates of postinfectious hydrocephalus $(\mathrm{PIH})$ and spina bifida when compared with HICs. ${ }^{9}$ Currently, no countryspecific data exist from Zambia about the burden of hydrocephalus, and most estimates are inferred from nearby African countries, such as Uganda and Tanzania..$^{10,11}$

The poor outcomes of this disease in LMICs are largely attributable to limited access to care ${ }^{12}$ and low literacy rates, ${ }^{13}$ but may also be due in part to the high PIH rates since neonatal meningitis is known to cause poor neurocognitive outcomes. ${ }^{14}$ Limited access to care is particularly detrimental for children with ventriculoperitoneal (VP) shunts, as these patients decline quickly when the device malfunctions..$^{15}$ This starkly contrasts with the typically more insidious symptoms of endoscopic third ventriculostomy and choroid plexus cauterization (ETV/CPC) failure. ${ }^{16}$ Knowledge of the limitations of shunt management in LMICs inspired the reintroduction of ETV/CPC in Uganda, a practice that was then adopted in other countries throughout the region and, ultimately, the world. ${ }^{17,18}$ This study describes patient demographics, surgical outcomes, and predictors of morbidity and mortality following surgical management of pediatric hydrocephalus at the major neurosurgical tertiary referral centers in Lusaka, Zambia.

\section{Methods \\ Patient Population}

This retrospective cohort study identified pediatric patients younger than 18 years who underwent surgical treatment for hydrocephalus at the University Teaching Hospital and the Beit-CURE Hospital in Lusaka, Zambia, between August 2017 and May 2019. These two centers treat and care for an estimated $90 \%$ of all pediatric hydrocephalus cases in Zambia. Patient referrals can be made to these two facilities by all physicians in Zambia. Although based in the capital city of Lusaka, these two hospitals serve as the country's only centers that provide specialized pediatric neurosurgical care. Since the Zambian government subsidizes healthcare, any patient who is a Zambian National Registration Card holder will receive surgical care free of charge with the exception of imaging, laboratory tests, and surgical implants. Surgical procedures performed included VP shunt insertions, VP shunt revisions, ETV with or without CPC, and ETV revisions. Patients who had multiple hydrocephalus proce- dures within the study window were counted only once; their first procedure in the study period was considered the index operation.

\section{Study Variables}

Data extraction was performed for the following study variables: age, sex, rural home province (as defined by population density according to the Zambian Census ${ }^{19}$ ), HIV status of the mother, hydrocephalus etiology, clinical symptomatology, head circumference at time of surgery (in centimeters), type of surgical intervention (VP shunt or ETV/CPC), and postoperative length of stay (in days). Two primary outcomes included 90-day mortality (as determined by the death certificate on the hospital registry or postoperative phone call if the patient died at home) and postoperative complications, which were treated as binary variables. Ninety-day complications included infection, wound dehiscence, CSF leak, readmission, and reoperation for any cause related to the index procedure. Patients could have more than one type of postoperative complication but were counted as having "any complication" only once. The time to primary outcomes was also extracted.

This study was approved by the CURE-International Hospitals, the Vanderbilt University Medical Center Institutional Review Board, the University of Zambia Biomedical Research Ethics Committee, and the Zambian National Health Research Authority. Data were manually collected from paper records and maintained in a password-protected REDCap (Research Electronic Data Capture) database for secure data storage purposes. ${ }^{20}$

\section{Statistical Analysis}

Statistical analysis was completed with $\mathrm{R}$ version 3.6.0 (R Foundation; https://www.r-project.org/). Continuous variables are presented as means with standard error estimates or as medians with IQRs if the data were nonnormally distributed. A descriptive analysis was performed comparing the association between outcome variables and surgical risk factors using the Wilcoxon test and Pearson test for continuous and categorical variables, respectively. Two additional subgroup analyses were conducted: 1) all patients whose index procedure was a primary surgery (not revision); and 2) infants younger than 12 months whose index procedure was a primary surgery. Odds ratios are presented with $95 \%$ CIs. Statistical significance was set a priori at $\mathrm{p}<0.05$. Variables with $\mathrm{p}<0.10$ on univariable analysis were included in multivariable binary logistic regression models for mortality and complications.

\section{Results}

\section{Patient Demographics}

A total of 378 patients met the inclusion criteria. The median age at the time of hydrocephalus surgery was 5.5 (IQR 3.1, 12.7) months. Forty-nine percent were male (n $=183)$, and $69 \%$ were from a rural province $(\mathrm{n}=259)$. The most common etiology of hydrocephalus was PIH, which accounted for $65 \%$ of the patient population $(\mathrm{n}=$ 226). Of the $35 \%$ of children with hydrocephalus attributable to noninfectious causes $(\mathrm{n}=121), 29 \%$ had congenital idiopathic hydrocephalus, $26 \%$ had myelomeningocele 
$(\mathrm{n}=32), 26 \%$ had tumor-associated hydrocephalus, and the remaining $19 \%$ were due to other causes such as intraventricular hemorrhage, congenital malformations, or aqueductal stenosis. The average head circumference was $5.3 \pm 5.2 \mathrm{~cm}$ above the age- and sex-adjusted 97th percentile on WHO charts; $42 \%(n=111)$ were more than 6 $\mathrm{cm}$ above the 97 th percentile. The most common presenting symptoms were irritability and changes in extraocular movements (65\% and 54\%, respectively). A summary of patient characteristics is shown in Table 1.

Of the 378 index procedures performed, there were $45 \mathrm{VP}$ shunt revisions, $281 \mathrm{VP}$ shunt insertions, $50 \mathrm{ETV} /$ $\mathrm{CPCs}$, and $1 \mathrm{ETV} / \mathrm{CPC}$ revision. The median postprocedural length of stay was $3(\mathrm{IQR} 2,4)$ days, and the median follow-up duration was 33 (IQR 4, 117) days. The 90-day postoperative mortality rate was $7 \%(\mathrm{n}=26)$, and the 90 day complication rate was $20 \%(\mathrm{n}=76)$. Of those with complications, the most common reported were reoperation (n $=44,58 \%)$ and infection $(\mathrm{n}=29,38 \%)$. The most common reason for reoperation was shunt removal for infection $(\mathrm{n}=$ 21, 48\%), followed by shunt insertion for ETV/CPC failure $(\mathrm{n}=8,18 \%)$. The mean time from surgical intervention to clinical complication was $29 \pm 27$ days (Table 1).

\section{First-Time Hydrocephalus Surgery Demographics}

Table 2 summarizes the subgroup analysis for patients who did not undergo revision as their index procedure, which included $82 \%(\mathrm{n}=309)$ of the total population. VP shunt insertions were more common $(n=264,85 \%)$ than ETV/CPCs $(\mathrm{n}=45,15 \%)$. This subgroup tended to be younger than the revision cohort at time of surgery at 5 (IQR 3, 9) months versus 56 (IQR 19, 134) months (p < $0.001)$. Patients who underwent revision were less likely to come from a rural province $(46 \%$ vs $72 \%, p<0.001)$. Those who had a prior hydrocephalus surgery presented with different symptoms of raised intracranial pressure than those who had not. The revision group was more likely to present with vomiting $(\mathrm{p}<0.001)$, seizures $(\mathrm{p}=$ $0.029)$, and failure to thrive ( $\mathrm{p}=0.013)$, while those $\mathrm{pa}-$ tients presenting with a new diagnosis of hydrocephalus were more likely to present with irritability $(\mathrm{p}<0.001)$ and changes in extraocular movements $(p<0.001)$. There was no difference in 90-day mortality after a revision operation compared with a primary shunt insertion or ETV/ CPC ( $p=0.369)$, but revision operations were associated with a higher complication rate $(\mathrm{p}=0.018)$.

An additional subgroup analysis was performed for infantile hydrocephalus, comparing children younger than 12 months of age who underwent VP shunt insertion versus ETV/CPC as their index procedure (Table 3). Sixteen percent of infants underwent ETV/CPC as their primary index procedure, which is similar to that of the entire cohort. Infants who underwent ETV/CPC were less likely to be from a rural province $(p=0.01)$ and were more likely to have two or more surgeons scrubbed into the procedure $(\mathrm{p}$ $=0.003$ ). All other analyzed variables showed no statistically significant difference in this subgroup.

\section{Univariable Analysis}

A summary of the univariable analysis for 90 -day com-
TABLE 1. Patient demographics and characteristics among a pediatric population from Zambia with hydrocephalus $(n=378)$

\begin{tabular}{|c|c|}
\hline & Value \\
\hline Median age at index op, mos (IQR) & $5.5(3.1,12.7)$ \\
\hline \multicolumn{2}{|l|}{ Sex, $n(\%)$} \\
\hline Male & $183(49)$ \\
\hline Female & $193(51)$ \\
\hline \multicolumn{2}{|l|}{ Rural home province, $\mathrm{n}(\%)$} \\
\hline Yes & $259(69)$ \\
\hline No & $119(31)$ \\
\hline \multicolumn{2}{|l|}{ HIV+ mother, $\mathrm{n}(\%)$} \\
\hline Yes & $20(5)$ \\
\hline No & $358(95)$ \\
\hline \multicolumn{2}{|l|}{ Symptoms prior to op, $\mathrm{n}(\%)$} \\
\hline Vomiting & $56(15)$ \\
\hline Lethargy & $78(21)$ \\
\hline Irritability & $247(65)$ \\
\hline Failure to thrive & $44(12)$ \\
\hline Seizures & $66(17)$ \\
\hline Oculomotor abnormality & $204(54)$ \\
\hline \multicolumn{2}{|l|}{ Hydrocephalus etiology, $n(\%)^{*}$} \\
\hline $\mathrm{PIH}$ & $226(65)$ \\
\hline Non-PIH hydrocephalus & $121(35)$ \\
\hline Congenital idiopathic & $35(29)$ \\
\hline MMC & $32(26)$ \\
\hline Tumor & $32(26)$ \\
\hline Encephalocele & $10(8)$ \\
\hline Aqueductal stenosis & $6(5)$ \\
\hline Cyst & $2(2)$ \\
\hline Dandy-Walker & $1(1)$ \\
\hline Posthemorrhagic & $0(0)$ \\
\hline Other & $4(3)$ \\
\hline \multicolumn{2}{|l|}{ MMC, n (\%) } \\
\hline Yes & $32(8)$ \\
\hline No & $346(92)$ \\
\hline \multicolumn{2}{|l|}{ 1st hydrocephalus op, $\mathrm{n}(\%) \dagger$} \\
\hline Yes & $309(87)$ \\
\hline No & $48(13)$ \\
\hline $\begin{array}{l}\text { Mean } \mathrm{cm} \text { above } 97 \text { th head circumference percentile } \\
( \pm \text { SE) }\end{array}$ & $5.3(5.2)$ \\
\hline \multicolumn{2}{|l|}{$\begin{array}{l}\text { Head circumference at op (cm above 97th percen- } \\
\text { tile), } n(\%)\end{array}$} \\
\hline$\leq 97$ th & $41(15)$ \\
\hline $0.01-2 \mathrm{~cm}$ above 97 th & $33(12)$ \\
\hline $2.01-4 \mathrm{~cm}$ above 97 th & $47(18)$ \\
\hline $4.01-6 \mathrm{~cm}$ above 97 th & $33(12)$ \\
\hline$>6 \mathrm{~cm}$ above 97 th & $111(42)$ \\
\hline \multicolumn{2}{|l|}{ Type of op performed, $\mathrm{n}(\%) \ddagger$} \\
\hline ETVICPC & $51(14)$ \\
\hline New VPS insertion & $281(75)$ \\
\hline VPS revision & $45(12)$ \\
\hline \multicolumn{2}{|l|}{ No. of surgeons scrubbed into case, $n(\%)$} \\
\hline 1 & $136(36)$ \\
\hline$>1$ & $242(64)$ \\
\hline Median postop LOS, days (IQR) & $3(2,4)$ \\
\hline Median postop follow-up duration, days (IQR) & $33(4,117)$ \\
\hline
\end{tabular}


» CONTINUED FROM PAGE 626

TABLE 1. Patient demographics and characteristics among a pediatric population from Zambia with hydrocephalus $(n=378)$

\begin{tabular}{lc}
\hline & Value \\
\hline 90-day mortality, $\mathrm{n}(\%)$ & \\
Yes & $26(7)$ \\
No & $352(93)$ \\
\hline 90-day complication, $\mathrm{n}(\%)$ & \\
Any & $76(20)$ \\
Reop for any reason & $44(12)$ \\
Shunt removal for infection & 21 \\
Shunt insertion for ETV/CPC failure & 8 \\
Shunt revision for failure & 7 \\
Wound revision only & 7 \\
Craniotomy for brain abscess evacuation & 1 \\
Infection & $29(8)$ \\
Treatment failure & $22(6)$ \\
CSF leak & $22(6)$ \\
Hospital readmission & $10(3)$ \\
Wound dehiscence & $7(2)$ \\
\hline Mean time to complication, days $( \pm$ SE) & $29(27)$ \\
\hline
\end{tabular}

LOS = length of stay; $M M C=$ myelomeningocele; $V P S=V P$ shunt.

* Data were available for 348 patients.

† Data were available for 357 patients.

$\ddagger$ Data were available for 377 patients.

plications and mortality is shown in Tables 4 and 5 and demonstrates that patients who had any complication were more likely to have undergone a revision hydrocephalus surgery $\left(\chi^{2}=8.39 ; p=0.004\right)$. Specifically, patients undergoing shunt revision surgery as opposed to primary shunt insertion or ETV/CPC were more likely to have a complication $\left(\chi^{2}=7.98 ; \mathrm{p}=0.018\right)$. When plotted against time, patients undergoing shunt revisions experienced complications more quickly than those undergoing new shunt insertions $(p=0.001)$ but not ETV/CPCs $(p=0.079)$ (Fig. 1). The follow-up duration was longer for patients who had a complication as opposed to those who did not (45 [IQR 16, 93] vs 27 [IQR 3, 124] days; $\mathrm{F}=4.09 ; \mathrm{p}=0.044)$.

For univariable 90-day mortality analysis (Table 5), patients who presented with failure to thrive as part of their constellation of symptoms were more likely to die $\left(\chi^{2}=6.34\right.$; $\mathrm{p}=0.012$ ). Also, patients who had complications were more likely to die than those who did not $\left(\chi^{2}=81.2 ; \mathrm{p}<0.001\right)$.

All other variables in Tables 4 and 5, including patients' age, home region, sex, maternal HIV status, etiology of hydrocephalus, head circumference, number of surgeons in the case, and initial postoperative hospital length of stay, showed no statistically significant association with mortality or 90-day complications on univariable analysis.

\section{Multivariable Analysis}

Failure to thrive and complication history were the two variables that met criteria for inclusion in the multivariable analysis for mortality and were not dependent on time (Table 6). Multicollinearity was tested for between these two variables and not present; thus, the statistical analysis is valid. In this analysis, the odds of a patient dying who had any type of complication were 43 times higher than those who did not have a postoperative complication ( $\mathrm{p}<$ 0.001; OR 42.9 [95\% CI 12.4-149.1]). Failure to thrive was no longer independently significant.

The multivariable analysis for 90-day complications included three variables: first-time hydrocephalus surgery, HIV-positive mother, and follow-up duration. Since the variable "type of hydrocephalus surgery" included both shunt revisions and new insertions, it was not included in this analysis due to concerns for collinearity. Patients undergoing revision hydrocephalus surgery were more likely to experience a complication than those undergoing first-time CSF diversion ( $p=0.008$; OR 2.45 [1.26-4.76]). Follow-up duration and an HIV-positive mother were not significant.

\section{Discussion}

This study is the first published epidemiological overview of pediatric hydrocephalus treatment in Zambia. Our findings are consistent with research in neighboring countries. ${ }^{11,21}$ Specifically, PIH is the most common etiology of hydrocephalus, and there is a high incidence of myelomeningocele-related hydrocephalus as well. Most patients have poor access to neurosurgical care in addition to low healthcare literacy, ${ }^{12,13}$ which contribute to the common diagnosis of neglected hydrocephalus. ${ }^{22}$ ETV/ CPCs have become an option for surgical intervention for a select group of hydrocephalus patients; however, the procedure's utility is still in its early stages in Zambia due to frequent equipment failure. ${ }^{23}$ Postoperative complications are common within 90 days after hydrocephalus surgery, occurring in 1 in 5 patients in our cohort. Understanding the current state of the disease in Zambia is critical to improving the care for pediatric neurosurgical patients.

Hydrocephalus disproportionally affects LMICs, which is primarily attributable to the increased incidence of PIH, and these findings are consistent in Zambia. In this study, $65 \%$ of participants had a history of central nervous system infection, compared with a rate of $7 \%$ in the US.,24 The relative increased incidence of PIH in LMICs is likely due to the standard practice of home birth, ${ }^{25}$ lack of appropriate maternal perinatal antibiotic administration, ${ }^{26}$ poor access to prenatal care, ${ }^{27,28}$ and aversion to CSF sampling when indicated. ${ }^{29}$ Improving patient and physician understanding of the risks of neonatal meningitis and destigmatizing lumbar punctures are essential to improving the quality of care. In Zambia, patients and caregivers commonly refuse lumbar punctures because of an inadvertent association with ensuing mortality due to the procedure's historically common use in diagnosing AIDS-defining illness during the sub-Saharan Africa HIV epidemic. ${ }^{29}$ Continuing to address this stigma is critical for accurate diagnosis of a CSF infection, which could subsequently improve neurodevelopmental outcomes in patients with hydrocephalus. ${ }^{14}$

In addition, our findings of myelomeningocele-related hydrocephalus are similar to other sub-Saharan African countries. Zambia is among the 94 countries worldwide that do not fortify food with folic acid ${ }^{30}$ According to the World Bank, $57 \%$ of the population lives below the international poverty line. ${ }^{1}$ Poverty contributes to malnutrition, 
TABLE 2. Subgroup analysis comparing patients whose index procedure was a primary shunt insertion versus a revision $(n=357)$

\begin{tabular}{|c|c|c|c|}
\hline & Revision $(n=48)$ & Primary $(n=309)$ & $p$ Value \\
\hline Median age at index op, mos (IQR) & $56(19,134)$ & $5(3,9)$ & $<0.001$ \\
\hline Sex, $n(\%)^{*}$ & & & 0.447 \\
\hline Male & $25(53)$ & $146(47)$ & \\
\hline Female & $22(47)$ & $163(53)$ & \\
\hline Rural home province, $\mathrm{n}(\%)$ & & & $<0.001$ \\
\hline No & $26(54)$ & $86(28)$ & \\
\hline Yes & $22(46)$ & $223(72)$ & \\
\hline HIV+ mother, n (\%) & & & 0.086 \\
\hline No & $48(100)$ & $291(94)$ & \\
\hline Yes & $0(0)$ & $18(6)$ & \\
\hline \multicolumn{4}{|l|}{ Symptoms prior to op, $\mathrm{n}(\%)$} \\
\hline Vomiting & & & $<0.001$ \\
\hline No & $27(56)$ & $274(89)$ & \\
\hline Yes & $21(44)$ & $35(11)$ & \\
\hline Lethargy & & & 0.466 \\
\hline No & $36(75)$ & $246(80)$ & \\
\hline Yes & $12(25)$ & $63(20)$ & \\
\hline Irritability & & & $<0.001$ \\
\hline No & $30(62)$ & $92(30)$ & \\
\hline Yes & $18(38)$ & $217(70)$ & \\
\hline Failure to thrive & & & 0.013 \\
\hline No & $37(77)$ & $277(90)$ & \\
\hline Yes & $11(23)$ & $32(10)$ & \\
\hline Seizures & & & 0.029 \\
\hline No & $34(71)$ & $259(84)$ & \\
\hline Yes & $14(29)$ & $50(16)$ & \\
\hline Oculomotor abnormality & & & $<0.001$ \\
\hline No & $44(92)$ & $123(40)$ & \\
\hline Yes & $4(8)$ & $186(60)$ & \\
\hline $\mathrm{PIH}, \mathrm{n}(\%) \dagger$ & & & 0.049 \\
\hline No & $11(50)$ & $91(30)$ & \\
\hline Yes & $11(50)$ & $214(70)$ & \\
\hline MMC, $\mathrm{n}(\%)$ & & & 0.013 \\
\hline No & $43(90)$ & $300(97)$ & \\
\hline Yes & $5(10)$ & $9(3)$ & \\
\hline Mean $\mathrm{cm}$ above 97 th head circumference percentile $( \pm$ SE) & $5.6(6.1)$ & $5.2(5.3)$ & 0.93 \\
\hline Initial type of op performed, $\mathrm{n}(\%)$ & & & $<0.001$ \\
\hline ETV & $1(2)$ & $45(15)$ & \\
\hline VPS & $47(98)$ & $264(85)$ & \\
\hline No. of surgeons scrubbed into case, $n(\%)$ & & & 0.008 \\
\hline 1 & $25(52)$ & $100(32)$ & \\
\hline$>1$ & $23(48)$ & $209(68)$ & \\
\hline Median postop LOS, days (IQR) & $3(2,4)$ & $3(2,4)$ & 0.112 \\
\hline Median postop follow-up duration, days (IQR) & $22(7,110)$ & $33(4,117)$ & 0.836 \\
\hline 90-day mortality, $\mathrm{n}(\%)$ & & & 0.369 \\
\hline No & $43(90)$ & $288(93)$ & \\
\hline Yes & $5(10)$ & $21(7)$ & \\
\hline 90-day complication, $\mathrm{n}(\%)$ & & & 0.004 \\
\hline No & $31(65)$ & $255(83)$ & \\
\hline Yes & $17(35)$ & $54(17)$ & \\
\hline Mean days to complication ( \pm SE) & $15(11)$ & $32(28)$ & 0.047 \\
\hline
\end{tabular}

* Data were available for 47 patients in the revision group.

† Data were available for 22 patients in the revision group and 305 patients in the primary group. 
TABLE 3. Subgroup analysis comparing infants with hydrocephalus who underwent their first hydrocephalus surgery, VP shunt or ETV/CPC, before 12 months of age $(n=249)$

\begin{tabular}{|c|c|c|c|}
\hline & VP Shunt $(n=209)$ & ETV/CPC $(n=40)$ & $\mathrm{p}$ Value \\
\hline Median age at index op, mos (IQR) & $3.9(2.5,6.0)$ & $4.1(2.7,7.2)$ & 0.611 \\
\hline Sex, $n(\%)$ & & & 0.760 \\
\hline Male & $110(53)$ & $20(50)$ & \\
\hline Female & $99(47)$ & $20(50)$ & \\
\hline Rural home province, $\mathrm{n}(\%)$ & & & 0.01 \\
\hline No & $44(21)$ & $16(40)$ & \\
\hline Yes & $165(79)$ & $24(60)$ & \\
\hline HIV+ mother, $\mathrm{n}(\%)$ & & & 0.460 \\
\hline No & $195(93)$ & $36(90)$ & \\
\hline Yes & $14(7)$ & $4(10)$ & \\
\hline \multicolumn{4}{|l|}{ Symptoms prior to op, $\mathrm{n}(\%)$} \\
\hline Vomiting & & & 0.668 \\
\hline No & $197(94)$ & $37(92)$ & \\
\hline Yes & $12(6)$ & $3(8)$ & \\
\hline Lethargy & & & 0.095 \\
\hline No & $171(82)$ & $37(92)$ & \\
\hline Yes & $38(18)$ & $3(8)$ & \\
\hline Irritability & & & 0.549 \\
\hline No & $51(24)$ & $8(20)$ & \\
\hline Yes & $158(76)$ & $32(80)$ & \\
\hline Failure to thrive & & & 0.617 \\
\hline No & $193(92)$ & $36(90)$ & \\
\hline Yes & $16(8)$ & $4(10)$ & \\
\hline Seizures & & & 0.193 \\
\hline No & $177(85)$ & $37(92)$ & \\
\hline Yes & $32(15)$ & $3(8)$ & \\
\hline Oculomotor abnormality & & & 0.584 \\
\hline No & $64(31)$ & $14(35)$ & \\
\hline Yes & $145(69)$ & $26(65)$ & \\
\hline $\mathrm{PIH}, \mathrm{n}(\%)^{*}$ & & & 0.115 \\
\hline No & $48(23)$ & $14(35)$ & \\
\hline Yes & $159(77)$ & $26(65)$ & \\
\hline MMC, n (\%) & & & 0.680 \\
\hline No & $201(96)$ & $39(98)$ & \\
\hline Yes & $8(4)$ & $1(2)$ & \\
\hline $\begin{array}{l}\text { Mean } \mathrm{cm} \text { above } 97 \text { th head circumference } \\
\text { percentile }( \pm \mathrm{SE})\end{array}$ & $5.2(5.5)$ & $4.0(3.8)$ & 0.262 \\
\hline No. of surgeons scrubbed into case, $n(\%)$ & & & 0.003 \\
\hline 1 & $76(36)$ & $5(12)$ & \\
\hline$>1$ & $133(64)$ & $35(88)$ & \\
\hline Median postop LOS, days (IQR) & $3(2,4)$ & $2.5(2,3)$ & 0.076 \\
\hline Median postop follow-up duration, days (IQR) & $33(4,104)$ & $43(14,186)$ & 0.151 \\
\hline 90-day mortality, $\mathrm{n}(\%)$ & & & 0.668 \\
\hline No & $197(94)$ & $37(92)$ & \\
\hline Yes & $12(6)$ & $3(8)$ & \\
\hline 90-day complication, $\mathrm{n}(\%)$ & & & 0.339 \\
\hline No & $175(84)$ & $31(78)$ & \\
\hline Yes & $34(16)$ & $9(22)$ & \\
\hline Mean days to complication ( \pm SE) & $27(28)$ & $40(23)$ & 0.08 \\
\hline
\end{tabular}

* Data were available for 207 patients in the VP shunt group. 
TABLE 4. Univariable analysis for 90-day complications for the entire cohort

\begin{tabular}{|c|c|c|c|c|}
\hline & $\begin{array}{l}\text { No Complication } \\
\quad(n=302)\end{array}$ & $\begin{array}{l}\text { Any Complication } \\
\qquad(\mathrm{n}=76)\end{array}$ & Statistic & $\mathrm{p}$ Value \\
\hline Median age at index op, mos (IQR) & $4.6(2.4,10.8)$ & $5.3(2.1,19.0)$ & $F=0.3$ & 0.57 \\
\hline Sex, $n(\%)^{*}$ & & & $\chi^{2}=1.1$ & 0.31 \\
\hline Male & $150(50)$ & $33(43)$ & & \\
\hline Female & $150(50)$ & $43(57)$ & & \\
\hline Rural home province, $\mathrm{n}(\%)$ & & & $\chi^{2}=0.3$ & 0.57 \\
\hline Yes & $209(69)$ & $50(66)$ & & \\
\hline No & $93(31)$ & $26(34)$ & & \\
\hline HIV+ mother, $\mathrm{n}(\%)$ & & & $\chi^{2}=3$ & 0.08 \\
\hline Yes & $19(6)$ & $1(1)$ & & \\
\hline No & $283(94)$ & $75(99)$ & & \\
\hline \multicolumn{5}{|l|}{ Presenting symptom, $\mathrm{n}(\%)$} \\
\hline Vomiting & & & $\chi^{2}=1.83$ & 0.18 \\
\hline Yes & $41(14)$ & $15(20)$ & & \\
\hline No & $261(86)$ & $61(80)$ & & \\
\hline Lethargy & & & $\chi^{2}=0.17$ & 0.68 \\
\hline Yes & $61(20)$ & $17(22)$ & & \\
\hline No & $241(80)$ & $59(78)$ & & \\
\hline Irritability & & & $\chi^{2}=0.81$ & 0.37 \\
\hline Yes & $194(64)$ & $53(70)$ & & \\
\hline No & $108(36)$ & $23(30)$ & & \\
\hline Failure to thrive & & & $\chi^{2}=1.59$ & 0.21 \\
\hline Yes & $32(11)$ & $12(16)$ & & \\
\hline No & $270(89)$ & $64(84)$ & & \\
\hline Seizure & & & $\chi^{2}=0.85$ & 0.36 \\
\hline Yes & $50(17)$ & $60(79)$ & & \\
\hline No & $252(83)$ & $16(21)$ & & \\
\hline Oculomotor abnormality & & & $\chi^{2}=2.4$ & 0.12 \\
\hline Yes & $169(56)$ & $35(46)$ & & \\
\hline No & $133(44)$ & $41(54)$ & & \\
\hline Hydrocephalus etiology, $n(\%) \dagger$ & & & $\chi^{2}=1.14$ & 0.29 \\
\hline $\mathrm{PIH}$ & $188(66)$ & $38(59)$ & & \\
\hline Non-PIH & $95(34)$ & $26(41)$ & & \\
\hline History of MMC, $n(\%)$ & & & $\chi^{2}=0.94$ & 0.33 \\
\hline Yes & $25(8)$ & $9(12)$ & & \\
\hline No & 277 (92) & $67(88)$ & & \\
\hline 1st-time hydrocephalus op, $\mathrm{n}(\%) \ddagger$ & & & $\chi^{2}=8.39$ & 0.004 \\
\hline Yes & $255(89)$ & $54(76)$ & & \\
\hline No & $31(11)$ & $17(24)$ & & \\
\hline $\begin{array}{l}\text { Mean } \mathrm{cm} \text { above } 97 \text { th head } \\
\text { circumference percentile }( \pm \mathrm{SE})\end{array}$ & $5.4(5.3)$ & $4.8(5.2)$ & $F=0.4$ & 0.53 \\
\hline Type of hydrocephalus op, $\mathrm{n}(\%) \S$ & & & $\chi^{2}=7.98$ & 0.018 \\
\hline VPS revision & $29(10)$ & $16(21)$ & & \\
\hline VPS insertion & $232(77)$ & $49(64)$ & & \\
\hline ETV & $40(13)$ & $11(14)$ & & \\
\hline No. of surgeons, $n(\%)$ & & & $\chi^{2}=0.2$ & 0.66 \\
\hline 1 & $107(35)$ & $29(38)$ & & \\
\hline$>1$ & $195(65)$ & $47(62)$ & & \\
\hline Median hospital LOS, days (IQR) & $3(2,4)$ & $3(2,4)$ & $F=0.56$ & 0.46 \\
\hline Median follow-up duration, days (IQR) & $27(3,124)$ & $45(16,93)$ & $F=4.09$ & 0.044 \\
\hline
\end{tabular}


TABLE 5. Univariable analysis for mortality for the entire cohort

\begin{tabular}{|c|c|c|c|c|}
\hline & Alive $(n=352)$ & Died $(n=26)$ & Statistic & $\mathrm{p}$ Value \\
\hline Median age at index op, mos (IQR) & $5.5(3.1,12.4)$ & $7.2(2.7,24.3)$ & $F=0.38$ & 0.54 \\
\hline Sex, $n(\%)^{*}$ & & & $\chi^{2}=0.3$ & 0.58 \\
\hline Male & $169(48)$ & $14(54)$ & & \\
\hline Female & $181(52)$ & $12(46)$ & & \\
\hline Rural home province, $\mathrm{n}(\%)$ & & & $\chi^{2}=0.63$ & 0.43 \\
\hline Yes & $243(69)$ & $16(62)$ & & \\
\hline No & $109(31)$ & $10(38)$ & & \\
\hline HIV+ mother, $\mathrm{n}(\%)$ & & & $\chi^{2}=1.56$ & 0.21 \\
\hline Yes & $20(6)$ & $0(0)$ & & \\
\hline No & $332(94)$ & $26(100)$ & & \\
\hline \multicolumn{5}{|l|}{ Presenting symptom, $\mathrm{n}(\%)$} \\
\hline Vomiting & & & $\chi^{2}=1.51$ & 0.22 \\
\hline Yes & $50(14)$ & $6(23)$ & & \\
\hline No & $302(86)$ & $20(77)$ & & \\
\hline Lethargy & & & $\chi^{2}=0.67$ & 0.41 \\
\hline Yes & $71(20)$ & $7(27)$ & & \\
\hline No & $281(80)$ & $19(73)$ & & \\
\hline Irritability & & & $\chi^{2}=0.18$ & 0.67 \\
\hline Yes & $231(66)$ & $16(62)$ & & \\
\hline No & $121(34)$ & $10(38)$ & & \\
\hline Failure to thrive & & & $\chi^{2}=6.34$ & 0.012 \\
\hline Yes & $37(11)$ & $7(27)$ & & \\
\hline No & $315(89)$ & $19(73)$ & & \\
\hline Seizure & & & $\chi^{2}=0.06$ & 0.81 \\
\hline Yes & $61(17)$ & $21(81)$ & & \\
\hline No & $291(83)$ & $5(19)$ & & \\
\hline Oculomotor abnormality & & & $\chi^{2}=0.18$ & 0.67 \\
\hline Yes & $191(54)$ & $13(50)$ & & \\
\hline No & $161(46)$ & $13(50)$ & & \\
\hline Hydrocephalus etiology, $n(\%) \dagger$ & & & $\chi^{2}=1.82$ & 0.18 \\
\hline $\mathrm{PIH}$ & $214(66)$ & $12(52)$ & & \\
\hline Non-PIH & $110(24)$ & $11(48)$ & & \\
\hline History of MMC, $\mathrm{n}(\%)$ & & & $\chi^{2}=0.9$ & 0.34 \\
\hline Yes & $33(9)$ & $1(4)$ & & \\
\hline No & $319(91)$ & $25(96)$ & & \\
\hline 1st-time hydrocephalus op, $\mathrm{n}(\%) \ddagger$ & & & $\chi^{2}=0.81$ & 0.37 \\
\hline Yes & $288(87)$ & $21(81)$ & & \\
\hline No & $43(13)$ & $5(19)$ & & \\
\hline $\begin{array}{l}\text { Mean } \mathrm{cm} \text { above } 97 \text { th head } \\
\text { circumference percentile }( \pm \mathrm{SE})\end{array}$ & $5.2(5.2)$ & $6.5(5.7)$ & $F=0.92$ & 0.34 \\
\hline Type of hydrocephalus op, n (\%)§ & & & $\chi^{2}=1.43$ & 0.49 \\
\hline VPS revision & $40(11)$ & $5(19)$ & & \\
\hline VPS insertion & $263(75)$ & $18(69)$ & & \\
\hline ETV & $48(14)$ & $3(12)$ & & \\
\hline No. of surgeons, $n(\%)$ & & & $\chi^{2}=0.02$ & 0.89 \\
\hline 1 & $127(36)$ & $9(35)$ & & \\
\hline$>1$ & $225(64)$ & $17(65)$ & & \\
\hline Median hospital LOS, days (IQR) & $3(2,4)$ & $4(2,5)$ & $F=2.23$ & 0.13 \\
\hline Median follow-up duration, days (IQR) & $33(4,119)$ & $22(11,56)$ & $F=0.03$ & 0.85 \\
\hline Any complication, $\mathrm{n}(\%)$ & & & $\chi^{2}=81.2$ & $<0.001$ \\
\hline Yes & $53(15)$ & $23(88)$ & & \\
\hline No & $299(85)$ & $3(12)$ & & \\
\hline
\end{tabular}


" CONTINUED FROM PAGE 631

TABLE 5. Univariable analysis for mortality for the entire cohort

\begin{tabular}{lcccc}
\hline & Alive $(\mathrm{n}=352)$ & Died $(\mathrm{n}=26)$ & Statistic & p Value \\
\hline Complication, $\mathrm{n}(\%)$ & & & & \\
\hline Treatment failure & & & $\chi^{2}=1.67$ & 0.20 \\
$\quad$ Yes & $19(5)$ & $3(12)$ & & \\
No & $333(95)$ & $23(88)$ & & \\
\hline Infection & & & $\chi^{2}=14.6$ & $<0.001$ \\
$\quad$ Yes & $22(6)$ & $7(27)$ & & \\
No & $330(94)$ & $19(73)$ & & 0.022 \\
Wound dehiscence & $5(1)$ & $2(8)$ & & \\
Yes & $347(99)$ & $24(92)$ & & \\
No & $16(5)$ & $6(23)$ & & \\
CSF leak & $336(95)$ & $20(77)$ & & \\
Yes & & & & \\
No & $7(2)$ & $3(12)$ & & \\
Readmission & $345(98)$ & $23(88)$ & & \\
Yes & $37(11)$ & $7(27)$ & & \\
No & $315(89)$ & $19(73)$ & & \\
\hline Reop & & & & \\
Yes & & & & \\
No & & & & \\
\hline
\end{tabular}

* Data were available for 350 patients who were alive.

† Data were available for 324 patients who were alive.

$\ddagger$ Data were available for 331 patients who were alive and 23 patients who had died.

$\S$ Data were available for 351 patients who were alive.

folic acid deficiency, ${ }^{31}$ and the high rate of spina bifida in Zambia. The incidence of spina bifida in Zambia is unknown. Nine percent of patients had myelomeningocelerelated hydrocephalus in our study, which is likely an underestimate, given poor case ascertainment due to many suspected deaths before reaching a healthcare provider's attention. Underestimation is also likely due to the fact that operations for most infants with cervical and thoracic defects are deferred, given the propensity for complication and life-limiting nature of these high lesions. In addition to high perinatal mortality, these children can often be neglected by caregivers given the challenging socioeconomic environment and limited infrastructure for Zambian families to support children with neurodevelopmental disabilities. ${ }^{32}$ Current efforts are underway in collaboration between the Zambian Ministry of Health and prominent local food production companies to try to address folate deficiency in the population. In addition to food fortification, improper food storage is gaining attention as a probable cause for folate deficiency due to proliferation of mycotoxins, which are folate receptor antagonists..$^{33}$ Lowering the incidence of spina bifida in Zambia should decrease the incidence of myelomeningocele-associated hydrocephalus but may not reduce the overall incidence of hydrocephalus as the frequency of other etiologies could rise, such as posthemorrhagic hydrocephalus of prematurity. ${ }^{34}$

Once children are diagnosed with hydrocephalus in Zambia, it is common practice for them to receive one of two primary treatments: VP shunt or ETV (with or without $\mathrm{CPC})$. The treatment that the surgeons plan is based on the etiology of hydrocephalus and anatomical favorability, two determining factors in the ETV Success Score. ${ }^{35}$ However, preoperative surgical planning is often superseded by equipment availability, which is often limited due to a lack of sterile processing solution for the flexible endoscope. Equipment limitations due to endoscope maintenance significantly impact the medical decision-making for Zambian neurosurgeons as well as neurosurgeons in LMICs across the globe. ${ }^{36}$ As a result, $14 \%$ of all study patients and $16 \%$ of infants received endoscopic treatment for their hydrocephalus, and there was $1 \mathrm{ETV} / \mathrm{CPC}$ revision in an older child, a relatively low number. In our study, there was no difference in the mortality rate between patients who received a shunt versus an ETV/CPC in the total cohort or in either of the subgroup analyses, similar to other published studies. ${ }^{15}$ Complication rates were more common and occurred relatively earlier after revision shunt procedures compared with new shunt insertions and ETV/ CPCs, as illustrated in Fig. 1. The prolonged time to complication for ETV/CPC and new shunt insertions could be due to an open fontanelle, poor caregiver understanding of red flag failure symptoms, or a patient who is not yet shunt dependent.

Ninety-day benchmarks for complications and mortality were used in our study instead of the standard 30-day period because patients in Zambia often present in a delayed fashion. The mean time to first detection of a complication was $29 \pm 27$ days after surgery. There is only one government-subsidized neurosurgical referral center for the country, and transportation to the hospital is a significant 
Reynolds et al.

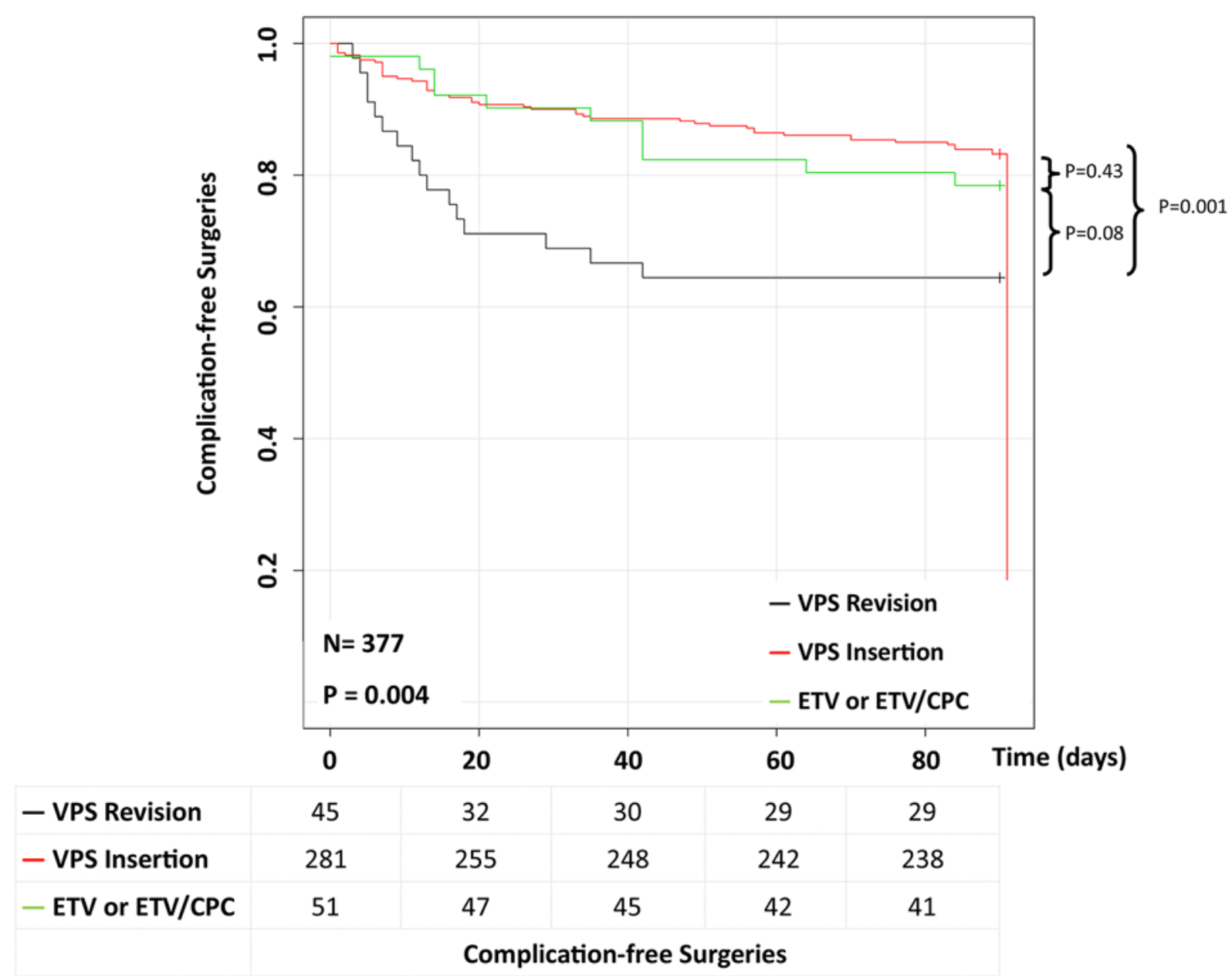

FIG. 1. Kaplan-Meier curve comparing time to postoperative complication for VP shunt (VPS) revision, VP shunt insertion, and ETVICPC procedures. Complications after VP shunt revision were more common and occurred more quickly than those after VP shunt insertions $(p=0.001)$. There was no statistical difference in time to complication between ETV/CPC and either procedure $(p=0.08, p=0.43)$. Figure is available in color online only.

financial burden for most families, especially those living outside the capital city of Lusaka. The high morbidity and mortality rates of the study population- $20 \%$ and $7 \%$, respectively-are consistent with those of other countries in sub-Saharan Africa. ${ }^{37}$ However, the mortality rate is higher than the $0.1 \%-3 \%$ reported in HICs. ${ }^{38,39}$ Prominent factors contributing to the higher complication rate in Zambia are

TABLE 6. Multivariable analysis for mortality and any complication for the entire cohort

\begin{tabular}{lcr}
\hline \multicolumn{1}{c}{ OR $(95 \% \mathrm{Cl})$} & p Value \\
\hline Mortality & & \\
\hline Failure to thrive & $3.02(0.97-9.42)$ & 0.056 \\
\hline Any complication & $42.91(12.35-149.13)$ & $<0.001$ \\
\hline Any complication & & \\
\hline HIV+ mother & $0.27(0.03-2.07)$ & 0.207 \\
\hline 1st-time hydrocephalus op & $2.45(1.26-4.76)$ & 0.008 \\
\hline Follow-up duration & $1.00(1.00-1.00)$ & 0.52 \\
\hline
\end{tabular}

the high reoperation and high infection rates. In the present study, $38 \%$ of the complications were due to infection, representing an overall infection risk of $8 \%$. Shunt studies completed in HICs have shown a potential benefit in reducing postoperative infections by using operative checklists ${ }^{40}$ and antibiotic-impregnated catheters. ${ }^{41}$ However, these expensive implants have not been demonstrated to be effective or economically feasible in LMICs. ${ }^{42}$ Thus, an emphasis on checklist compliancy, reducing overcrowding on the postoperative wards, and improving equipment are major focuses in Zambia. Furthermore, malnutrition is very prominent in Zambia. Malnutrition in other settings is a known predisposing factor to operative complications such as infection due to the creation of an immunocompromised state. ${ }^{43,44}$ Failure to thrive was common in this study cohort, but the true incidence of malnutrition was not available from the records and warrants further prospective research. Our study reinforces the need to provide means to reduce complications in hopes of reducing mortality and improving neurological outcomes.

Neurosurgical postoperative complications are a global point of discussion. To successfully manage complica- 
tions, a neurosurgeon must ensure adequate follow-up and family understanding of correct wound care and red flag symptoms at discharge. The average follow-up duration for our study was 33 (IQR 4, 117) days over a 22-month period. While this is a good foundation, $87 \%$ of the group had a VP shunt, which are known to be unpredictable in their failure patterns and require lifelong neurosurgical care. Thus, longer patient follow-up is a goal. Anecdotally, shortened follow-up duration is not due to the lack of emphasis for continued care by the treating surgeons, but more due to families being unable to afford transportation for long travel distances and high illiteracy rates compromising patient understanding of the condition and postsurgical recommendations. ${ }^{45}$ Education programs in the Zambian village languages are currently in development to help address misunderstandings.

\section{Study Limitations}

The limitations of this study are those inherent to a retrospective study. Electronic health records are not currently supported in Zambian public hospitals, so available data are confined to what is legible in each patient's paper chart that is carried on their person. The study is not a population-based study by design; however, it can be considered generalizable for the country, given the fact that the hospitals in this study support approximately $90 \%$ of pediatric neurosurgical services in Zambia. The group excluded from this population are the wealthy elite who are able to seek care in private facilities or in more developed neighboring countries. This skews the population toward a more impoverished, low literacy group. Yet, since the majority of the Zambian public lives in poverty, ${ }^{1}$ these numbers should be an accurate representation of the population receiving national healthcare. The study is also limited by the large number of patients who were lost to follow-up (33-day median follow-up), which could be a source of bias that leads to underrepresentation of outof-hospital mortality and 90-day complications. However, the interquartile range for follow-up extended up to 117 days, and if the complication window were limited to 30 days, too many outcomes would be missed (Fig. 1). Lastly, despite centralized healthcare, there is a degree of case ascertainment bias given that many children born at home with hydrocephalus may die prior to hospital evaluation. Future prospective, population-based studies with a longer follow-up duration are currently in progress to better understand the true incidence and clinical outcomes of pediatric hydrocephalus and spina bifida in Zambia.

\section{Conclusions}

Pediatric PIH is the most common etiology of hydrocephalus in Zambia. Neglected hydrocephalus is a common presentation, and most children undergo VP shunt insertion for surgical treatment of the disease. Postoperative complications affect 1 in 5 patients undergoing surgery for hydrocephalus and represent a significant predictor of patient mortality. Our continued evaluation of this patient population will 1) allow us to better understand and develop tools to reduce postoperative complications, including surgical site infections; 2) allow us to further understand the natural history and etiology of hydrocephalus in Zambia; and 3) provide care that will systematically change the overall health of children diagnosed with hydrocephalus in Zambia.

\section{Acknowledgments}

We thank the Fogarty International Center and the Vanderbilt University, Emory University, Cornell University, and Duke University (VECD) consortium for their project support. We also thank CURE International for their assistance with research materials and database maintenance.

Dr. Reynolds serves as a Fogarty Global Health Trainee affiliated with the Vanderbilt-Emory-Cornell-Duke Consortium and is supported by the Fogarty International Center at the National Institutes of Health (NIH) under grant no. R25TW009337. Dr. Garcia serves as a Fogarty Global Health Trainee and is supported by the Fogarty International Center under grant no. D43TW010543 through the Harvard University, Boston University, Northwestern University, and University of New Mexico consortium. The content is solely the responsibility of the authors and does not necessarily represent the official views of the NIH.

\section{References}

1. Poverty and Equity Data Portal. Zambia. World Bank. Accessed June 25, 2020. https://povertydata.worldbank.org

2. World Bank Country and Lending Groups. World Bank. Accessed June 25, 2020. https://datahelpdesk.worldbank.org/ knowledgebase/articles/906519-world-bank-country-andlending-groups

3. Global Neurosurgical Workforce Map. 2016 World Neurosurgery Workforce. World Federation of Neurosurgical Societies. Accessed June 26, 2020. https://www.wfns.org/menu/61/ global-neurosurgical-workforce-map

4. El Khamlichi A. African neurosurgery: current situation, priorities, and needs. Neurosurgery. 2001;48(6):1344-1347.

5. Sader E, Yee P, Hodaie M. Barriers to neurosurgical training in Sub-Saharan Africa: the need for a phased approach to global surgery efforts to improve neurosurgical care. World Neurosurg. 2017;98:397-402.

6. Abou-Hamden A, Drake J. Hydrocephalus and arachnoid cysts. In: Swaiman's Pediatric Neurology. Vol. 6. Elsevier; 2017.

7. Dewan MC, Rattani A, Mekary R, et al. Global hydrocephalus epidemiology and incidence: systematic review and metaanalysis. J Neurosurg. 2019;130(4):1065-1079.

8. Isaacs AM, Riva-Cambrin J, Yavin D, et al. Age-specific global epidemiology of hydrocephalus: systematic review, metanalysis and global birth surveillance. PLoS One. 2018; 13(10):e0204926.

9. Warf BC. Hydrocephalus in Uganda: the predominance of infectious origin and primary management with endoscopic third ventriculostomy. J Neurosurg. 2005;102(1)(suppl):1-15.

10. Warf BC. Pediatric hydrocephalus in East Africa: prevalence, causes, treatments, and strategies for the future. World Neurosurg. 2010;73(4):296-300.

11. Santos MM, Rubagumya DK, Dominic I, et al. Infant hydrocephalus in sub-Saharan Africa: the reality on the Tanzanian side of the lake. J Neurosurg Pediatr. 2017;20(5):423-431.

12. Simpamba MM, Struthers PM, Mweshi MM. Access to health care for children with neural tube defects: experiences of mothers in Zambia. Afr J Disabil. 2016;5(1):267.

13. Zambia. The World Bank. Accessed June 26, 2020. https:// data.worldbank.org/country/zambia?view=chart

14. Edmond K, Clark A, Korczak VS, et al. Global and regional risk of disabling sequelae from bacterial meningitis: a systematic review and meta-analysis. Lancet Infect Dis. 2010; 10(5):317-328.

15. Kulkarni AV, Schiff SJ, Mbabazi-Kabachelor E, et al. Endo- 
scopic treatment versus shunting for infant hydrocephalus in Uganda. N Engl J Med. 2017;377(25):2456-2464.

16. Dewan MC, Lim J, Morgan CD, et al. Endoscopic third ventriculostomy with choroid plexus cauterization outcome: distinguishing success from failure. J Neurosurg Pediatr. 2016; 25(6):655-662.

17. Warf BC, Campbell JW. Combined endoscopic third ventriculostomy and choroid plexus cauterization as primary treatment of hydrocephalus for infants with myelomeningocele: long-term results of a prospective intent-to-treat study in 115 East African infants. J Neurosurg Pediatr. 2008;2(5): 310-316.

18. Dewan MC, Naftel RP. The global rise of endoscopic third ventriculostomy with choroid plexus cauterization in pediatric hydrocephalus. Pediatr Neurosurg. 2017;52(6):401-408.

19. Zambia Statistics Agency. Census of Population Summary Report. Zambian Central Statistics Office. Accessed June 25, 2020. https://www.zamstats.gov.zm/index.php/publications/ category/14-2010

20. Harris PA, Taylor R, Thielke R, et al. Research electronic data capture (REDCap) - a metadata-driven methodology and workflow process for providing translational research informatics support. J Biomed Inform. 2009;42(2):377-381.

21. Gathura E, Poenaru D, Bransford R, Albright AL. Outcomes of ventriculoperitoneal shunt insertion in Sub-Saharan Africa. J Neurosurg Pediatr. 2010;6(4):329-335.

22. Garg K, Gurjar HK, Singh PK, et al. Can a head get bigger than this? Report of a neglected case of hydrocephalus. Asian J Neurosurg. 2014;9(4):233-234.

23. Cheelo M, Brugha R, Bijlmakers L, et al. Surgical capacity at district hospitals in Zambia: from 2012 to 2016. World J Surg. 2018;42(11):3508-3513.

24. Stone SS, Warf BC. Combined endoscopic third ventriculostomy and choroid plexus cauterization as primary treatment for infant hydrocephalus: a prospective North American series. J Neurosurg Pediatr. 2014;14(5):439-446.

25. Haddad DN, Makin JD, Pattinson RC, Forsyth BW. Barriers to early prenatal care in South Africa. Int J Gynaecol Obstet. 2016;132(1):64-67.

26. Bang AT, Bang RA, Baitule SB, et al. Effect of home-based neonatal care and management of sepsis on neonatal mortality: field trial in rural India. Lancet. 1999;354(9194):1955-1961.

27. Susuman AS, Chialepeh WN, Bado A, Lailulo Y. High infant mortality rate, high total fertility rate and very low female literacy in selected African countries. Scand J Public Health. 2016;44(1):2-5.

28. Wong KLM, Benova L, Campbell OMR. A look back on how far to walk: systematic review and meta-analysis of physical access to skilled care for childbirth in Sub-Saharan Africa. PLoS One. 2017;12(9):e0184432.

29. Thakur KT, Mateyo K, Hachaambwa L, et al. Lumbar puncture refusal in sub-Saharan Africa: a call for further understanding and intervention. Neurology. 2015;84(19): 1988-1990.

30. Darnton-Hill I. Fortification of wheat flour. Biological, behavioural and contextual rationale. World Health Organization. e-Library of Evidence for Nutrition Actions (eLENA). Published July 2017. Accessed June 25, 2020. https://www. who.int/elena/titles/bbc/wheat-flour-fortification/en/

31. Simuyemba MC, Bwembya PA, Chola M, Michelo C. A root cause analysis of sub-optimal uptake and compliance to iron and folic acid supplementation in pregnancy in 7 districts of Zambia. BMC Pregnancy Childbirth. 2020;20(1):20.

32. Chiluba BC, Moyo G. Caring for a cerebral palsy child: a caregivers perspective at the University Teaching Hospital, Zambia. BMC Res Notes. 2017;10(1):724.

33. Du RY, LoPresti MA, Hadley CC, Lam S. Systematic review of clinician awareness of mycotoxin impact in neural tube defects and best practices for pediatric neurosurgeons: impli- cations for public health and policy. Childs Nerv Syst. 2019; 35(4):637-644.

34. Radic JA, Vincer M, McNeely PD. Temporal trends of intraventricular hemorrhage of prematurity in Nova Scotia from 1993 to 2012. J Neurosurg Pediatr. 2015;15(6):573-579.

35. Kulkarni AV, Drake JM, Mallucci CL, et al. Endoscopic third ventriculostomy in the treatment of childhood hydrocephalus. J Pediatr. 2009;155(2):254-259.e1.

36. Santos MM, Qureshi MM, Budohoski KP, et al. The growth of neurosurgery in East Africa: challenges. World Neurosurg. 2018;113:425-435.

37. Reid T, Grudziak J, Rodriguez-Ormaza N, et al. Complications and 3-month outcomes of children with hydrocephalus treated with ventriculoperitoneal shunts in Malawi. J Neurosurg Pediatr. 2019;24(2):120-127.

38. Wu Y, Green NL, Wrensch MR, et al. Ventriculoperitoneal shunt complications in California: 1990 to 2000. Neurosurgery. 2007;61(3):557-563.

39. Smith ER, Butler WE, Barker FG II. In-hospital mortality rates after ventriculoperitoneal shunt procedures in the United States, 1998 to 2000: relation to hospital and surgeon volume of care. J Neurosurg. 2004;100(2 Suppl Pediatrics): 90-97.

40. Kestle JR, Riva-Cambrin J, Wellons JC III, et al. A standardized protocol to reduce cerebrospinal fluid shunt infection: the Hydrocephalus Clinical Research Network Quality Improvement Initiative. J Neurosurg Pediatr. 2011;8(1):22-29.

41. Mallucci CL, Jenkinson MD, Conroy EJ, et al. Antibiotic or silver versus standard ventriculoperitoneal shunts (BASICS): a multicentre, single-blinded, randomised trial and economic evaluation. Lancet. 2019;394(10208):1530-1539.

42. Lane JD, Mugamba J, Ssenyonga P, Warf BC. Effectiveness of the Bactiseal Universal Shunt for reducing shunt infection in a sub-Saharan African context: a retrospective cohort study in 160 Ugandan children. J Neurosurg Pediatr. 2014; 13(2):140-144.

43. Moramarco S, Amerio G, Kasengele Chipoma J, et al. Filling the gaps for enhancing the effectiveness of community-based programs combining treatment and prevention of child malnutrition: results from the Rainbow Project 2015-17 in Zambia. Int J Environ Res Public Health. 2018;15(9):E1807.

44. Tobert CM, Hamilton-Reeves JM, Norian LA, et al. Emerging impact of malnutrition on surgical patients: literature review and potential implications for cystectomy in bladder cancer. J Urol. 2017;198(3):511-519.

45. Pugliese OT, Solari JL, Ferreres AR. The extent of surgical patients' understanding. World J Surg. 2014;38(7):1605-1609.

\section{Disclosures}

The authors report no conflict of interest concerning the materials or methods used in this study or the findings specified in this paper.

\section{Author Contributions}

Conception and design: Reynolds, Bhebhe. Acquisition of data: Reynolds. Analysis and interpretation of data: Reynolds, Zhao. Drafting the article: Reynolds. Critically revising the article: Reynolds, Bhebhe, Garcia, Lam, Sichizya, Shannon. Reviewed submitted version of manuscript: Reynolds, Bhebhe, Garcia, Lam, Sichizya, Shannon. Approved the final version of the manuscript on behalf of all authors: Reynolds. Statistical analysis: Zhao. Study supervision: Sichizya, Shannon.

\section{Correspondence}

Rebecca A. Reynolds: Vanderbilt University Medical Center, Nashville, TN. rebecca.a.kasl@vumc.org. 